\title{
CONTAMINATION RESISTS IN METASTABLE ATOM LITHOGRAPHY
}

\author{
M. Baker, A.J. Palmer and R.T. Sang \\ Laser Atomic Physics Laboratory, Centre for Quantum Dynamics, \\ Griffith University, Nathan, QLD 4111 Australia
}

\begin{abstract}
We have used a metastable argon beam to expose gold-coated silicon substrates covered with a self assembled monolayer (SAM) resist. The substrates have been covered with a patterned mask, with features of $10 \mu \mathrm{m}$ size, and exposed to the atomic beam. Subsequent etching revealed negative contrast patterns, consistent with the formation of a negative contamination resist in the SAM, which we attribute to background pump oil vapour. Metastable dosages of $9 \times 10^{14}$ atoms $\mathrm{cm}^{-2}$ and exposure times $<1 \mathrm{hr}$ have been sufficient to produce reliable negative resists.
\end{abstract}

\section{INTRODUCTION}

Atom lithography, which utilises a beam of neutral atoms as a lithography tool, is one area of interest currently being pursued to create nanoscale structures [1]. The impetus for this field has come from the need for lithographic techniques that will produce smaller features and finer resolution than existing optical lithographic methods. Current optical lithographic techniques are diffraction limited to resolutions above $\lambda / 2$, where $\lambda$ is the wavelength of the light source used. The use of atomic beams reduces this diffraction limit, since the de Broglie wavelength associated with an atom is very small compared to the wavelengths of existing light sources. The neutral charge of the atoms means no space charge effects exist to cause beam divergence and reduce resolution (as occurs in charged particle lithography). Finally, their laser accessible internal states allows manipulation of atomic beam with optical forces to create arbitrary patterns [2].

One of the most promising candidates for neutral atom lithography lies in the application of excited-state metastable Noble gas atoms [3]. These metastable states are long lived ( $>1 \mathrm{~s})$ energy levels that lie far above (10-20 eV) the ground state. Upon collision with a surface, the metastable atom undergoes de-excitation and delivers this energy in a very small area on the surface. With the use of an appropriate resist layer on the surface, arbitrary patterns may be produced, and transferred to the underlying substrate via etching processes.

The nature of the resist layer is of significant importance in order to create nanometer size structures faithfully with the desired resolution. Research with metastable atom lithography has focused on the application of long chain alkanethiols as a resist layer. These long chain hydrocarbons contain a reactive sulphur head group that bonds to the gold substrate to form an ordered self assembled hydrophobic monolayer (SAM). The wetting properties of the SAM change when damaged by a metastable atom or other energetic particle. Their high density $\sim 4.6 \times 10^{14}$ mole $\mathrm{cm}^{-2}$, and small thickness $\sim 1.5 \mathrm{~nm}$ make them ideal for application as a resist layer [4].

Metastable atomic beams of argon, helium, and neon [4-7] have all been used to pattern various resist covered substrates. SAM covered substrates have been used to create positive patterns. In addition, negative patterning has been observed in SAM covered substrates, as well as substrates exposed in the presence of a thin adsorbed layer of background pump oil on the substrate [8,9]. In both of these cases, the negative resists formed have been attributed to the interaction between the metastable atom with the thin contamination film, to form a durable carbon-based material resistant to the subsequent etching steps. For the case of argon, high dosage levels and long exposure times have been required in order to form a durable contamination resist. Previous work has found that a dosage of $\sim 1 \times 10^{16}$ atoms $\mathrm{cm}^{-2}$ is required to sufficiently damage the SAM layer for the subsequent etching step [13]. Such large dosages correspond to exposure times of many hours.

This letter reports on the formation of negative resist formation in SAM covered substrates exposed to a metastable beam of argon. The negative resist layer formed requires smaller metastable dosage and exposure times than observed previously for metastable argon. Furthermore, evidence is provided to support the nature of the resist as the interaction between the metastable atoms and a thin adsorbed contamination layer of background pump oil. 


\section{SETUP}

The metastable argon beam is produced in a DC discharge source, described elsewhere [10]. A discharge is created between the cathode pin and the skimmer plate as the argon atoms expand supersonically from an insulated boron nitride nozzle. Electron collision with the atoms excites a proportion of ground state atoms into the metastable state, with energy of $10 \mathrm{eV}$. The metastable beam then passes through the skimmer hole and into the sample chamber. The source chamber is pumped by a diffusion pump, base pressure $2 \times 10^{-7}$ Torr which rises to $2 \times 10^{-4}$ Torr when the source is operational. The sample chamber is evacuated by a turbo pump which has a base pressure of $2 \times 10^{-7}$ Torr, and rises to $1 \times 10^{-5}$ Torr when the metastable beam is activated. A schematic of the system is provided below (see Fig.1). The average flux for Ar is $2.5 \times 10^{14}$ at a discharge current of $15 \mathrm{~mA}$ [10]

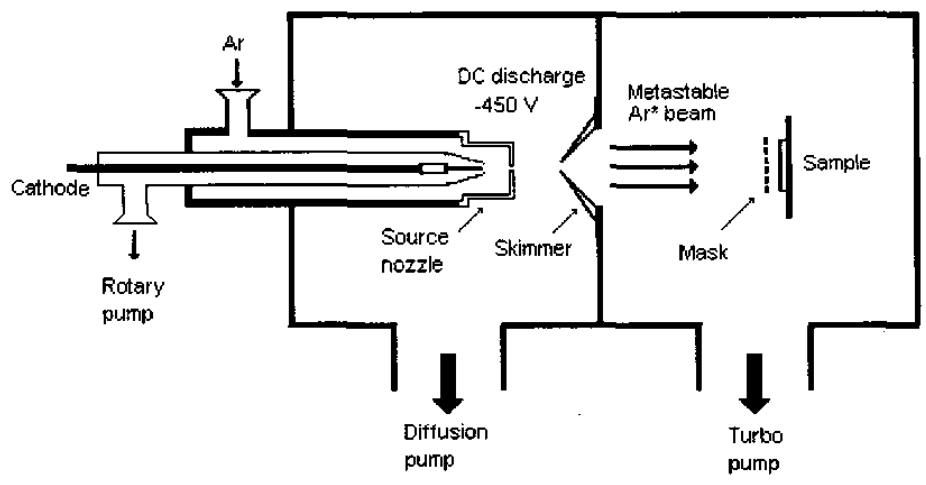

Figure 1 - Schematic of the metastable atom beam lithography apparatus.

\section{ATOM LITHOGRPAHY EXPOSURES}

The wafers used were $<1 \quad 00>$ test grade silicon $(\sim 400 \mu \mathrm{m}$ thick, 4" diameter). These wafers were cleaved into smaller $25 \mathrm{~mm} \times 25 \mathrm{~mm}$ sections. The substrates were sputtered with thin layers of $\mathrm{Cr}(\sim 3 \mathrm{~nm})$ and $\mathrm{Au}(40 \mathrm{~nm})$. The thin $\mathrm{Cr}$ layer improves the adherence of $\mathrm{Au}$ to the silicon substrate. The rms surface roughness of the $\mathrm{Au}$ surface was measured with an AFM to be $\sim 2 \mathrm{~nm}$.

To form the SAM resist layer, the substrates were placed into a $\sim 2 \mathrm{mM}$ solution of dodecanethiol $\mathrm{CH}_{3}\left(\mathrm{CH}_{2}\right)_{11} \mathrm{SH}$ in ethanol, for a period of $\sim 20$ hours [11]. The substrates were then removed from the solution, rinsed in ethanol, and blown dry with nitrogen gas. In order to test the quality of the SAM layer, contact angle measurements were conducted with $2 \mu \mathrm{L}$ water droplets placed onto the substrate. These yielded contact angles $>110$, indicating a high quality SAM [11]. In addition, ellipsometry conducted on samples removed from the SAM solution indicated the presence of $\mathrm{a} \sim 13 \quad 5 \AA$ thick layer, confirming the formation of the SAM upon the gold substrate [12]. This is in close agreement with the expected thickness of $18-21 \AA[11]$

A set of patterned transmission electron microscopy (TEM) copper masks were placed over the substrates, with typical feature size of $\sim 10 \mu \mathrm{m}$. The substrates were mounted onto a specially designed sample holder, and placed into the sample chamber at a distance $27 \mathrm{~cm}$ from the skimmer. The system was evacuated, and the samples exposed to the metastable beam for various periods to investigate the required dosage to create reliable patterns. The typical flux at the position of the samples was $\sim 3.5 \times 10^{11} \mathrm{~s}^{-1} \mathrm{~cm}^{-2}$ which was measured with a stainless steel plate Faraday cup able to be positioned in the beam.

After exposure to the beam, the samples were removed from the system and placed into a ferrocyanide etching solution, consisting of $\mathrm{KOH}(1 \mathrm{M}), \mathrm{K}_{2} \mathrm{~S}_{2} \mathrm{O}_{3}(0.1 \mathrm{M}), \mathrm{K}_{3} \mathrm{Fe}(\mathrm{CN})_{6}(0.01 \mathrm{M})$ and $\mathrm{K}_{4} \mathrm{Fe}(\mathrm{CN})_{6}(0.001 \mathrm{M})$ [13]. The samples were removed periodically from the solution and inspected during the process. A number of samples were exposed to investigate the effect of metastable dosage, exposure time, and optimum etch time on the pattern formation. Unexpectedly, the patterns produced all formed the negative patterns, rather than the positive 
patterns expected, indicating the formation of a negative resist in the SAM layer in the areas exposed by the metastable atoms.

The best negative patterns occurred for samples exposed to a metastable dosage of $9 \times 10^{14} \mathrm{~cm}^{-2}$, with an exposure time of 45 minutes. An etch time of 10 minutes was found to give the most reproducible results. This dosage corresponds to $\sim 2 \mathrm{Ar}^{*}$ atoms for every SAM molecule. Negative patterns were also observed for smaller dosage levels, but the resulting etch step resulted in poor patterning in the substrate. Samples were analysed with atomic force microscopy (contact mode) to determine the quality of the pattern and etch depth (see Fig. 2). The periodic line noise on the left of images b) and c) is an artefact of the AFM.

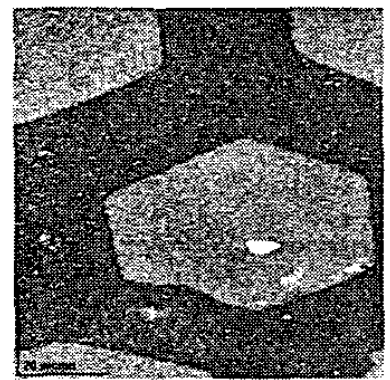

a)

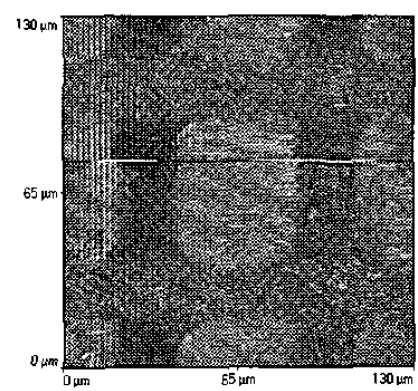

b)

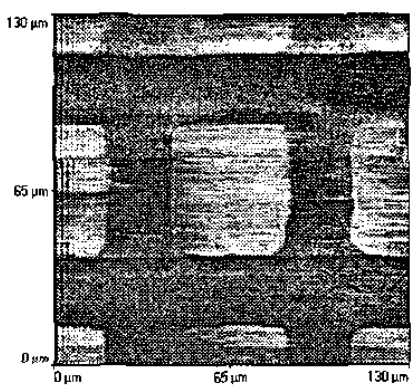

c)

Figure 2. AFM images of negative pattern formation in Au coated Si substrates

a) Surface of a hexagonal mesh pattern of Au on silicon - $80 \mu \mathrm{m} \times 80 \mu \mathrm{m}$. The two regions are $\mathrm{Au}$ (bright area), and the surrounding etched silicon (dark area) corresponding to an etch depth of $\sim 60 \mathrm{~nm}$ (JEOL JSPM-4200)

b) Negative pattern on the surface of a silicon square mesh pattern $-130 \mu \mathrm{m} \times 130 \mu \mathrm{m}$, etch depth of $40 \mathrm{~nm}$. (ThermoMicroscopes TMX 2000 Explorer)

c) Lateral force image showing the presence of the residual resist layer $-130 \mu \mathrm{m} \times 130 \mu \mathrm{m}$ (ThermoMicroscopes TMX 2000 Explorer)

The formation of negative resists has been attributed to contamination from background pump oil vapour in the system [8]. To confirm the presence of such a contamination resist in our system, a bare gold substrate was placed into the sample chamber, and exposed with a dosage of $\sim 8 \times 10^{14} \mathrm{~cm}^{-2}$, a similar dosage to that used on substrates with SAM resists. The etching revealed that a negative resist had formed in the areas exposed to the metastable beam, and the negative pattern was subsequently transferred into the underlying substrate. The contamination resist formed in this case was not particularly resilient to the etching solution, and from our preliminary exposures, it would appear that the metastable atom beam in conjunction with a SAM layer forms a more robust contamination resist.

As a further measure, a residual gas analyser (Stanford Research Systems RGA-100) with a mass range of 1 to $100 \mathrm{amu}$ was fitted to the sample chamber in order to verify the presence of contaminants such as pump oil vapours in the background vacuum of the sample chamber. The RGA detected the presence of significant pump oil contamination in the system, with a partial pressure of $1.5 \times 10^{-8}$ Torr, contributing $\sim 5 \%$ of the total background. The source of this contamination is most likely from the mechanical rotary pump and turbo pump used to evacuate the sample chamber. Although such uncontrolled contamination would normally be regarded as a hindrance, it would appear that in this case it is providing the mechanism for the rapid formation of a robust negative resist layer.

Previous groups have required substantial dosages of metastable atoms $\left(\sim 1 \times 10^{16}\right.$ atoms $\left.\mathrm{cm}^{-2}\right)$ and very long exposure periods ( 18 hours) in order to produce contamination resists and reliable patterns [8]. In contrast, we have produced reliable patterns with much smaller metastable dosages $\left(9 \times 10^{14}\right.$ atoms $\left.\mathrm{cm}^{-2}\right)$ and much shorter exposure times ( $<1$ hour) than previously required. 


\section{CONCLUSIONS}

This work has demonstrated the rapid formation of contamination resists on SAM covered substrates. The metastable dosage required to form the resist and provide a reliable pattern is $9 \times 10^{14}$ atoms $\mathrm{cm}^{-2}$ in a period less than an hour. These figures compare very well with the required metastable dosages of $\sim 1 \times 10^{16}$ atoms $\mathrm{cm}^{-2}$, and long exposure times ( $\sim 18$ hours) for contamination resists reported by other groups. The formation of the resist has been attributed to the presence of pump oil contamination in the vacuum system. The application of contamination resists offer great promise in further reducing the required metastable dosages and exposure time to generate reliable patterns, and make the process more viable for large scale application.

Our future work will aim to develop a more controlled method of contamination in order to optimise the formation of the resist, to characterise the nature of the contamination resist by various means (XPS, ellipsometry, contact angle goniometer), and observe it's formation using other metastable species.

\section{ACKNOWLEDGMENTS}

We would like to thank Greg Watson and Jola Blach for AFM analysis of the samples, and Ray Sweatman and Dennis Sweatman and the CRC for Microtechnology, for access to equipment for the preparation of substrates. The project was supported by a Griffith University Research and Development Grant and MB was supported by a Griffith University Postgraduate Research Award.

\section{REFERENCES:}

[1] K.K. Berggren, A. Bard, J.L. Wilbur, J.D. Gillaspy, A.G. Helg, J.J. McClelland, S.L. Rolston, W.D. Phillips, M. Prentiss and G.M. Whitesides, Science 269 1255, (1995)

[2] G. Timp, R.E. Behringer, D.M. Tennant, J.E. Cunningham, M. Prentiss, K.K. Berggren, Phys. Rev. Lett. 69, 1636, (1992)

[3] J.H. Thywissen, K.S. Johnson, R. Younkin, N.H. Dekker, K.K. Berggren, A.P. Chu, M. Prentiss, J. Vac. Sci. Technol. B 15 (6), (1997)

[4] A. Bard, K.K. Berggren, J.L. Wilbur, J.D. Gillapsy, S.L. Rolston, J.J. McClelland, W.D. Phillips, M. Prentiss, G.M. Whitesides, J. Vac. Sci. Technol. B 15 (5), (1997)

[5] A.S. Bell, B.Brezger, U.Drodofsky, S.Nowak, T. Pfau, J. Stuhler, Th. Schulze, J. Mlynek, Surface Science 433-435, 40-47, (1999)

[6] W. Lu, K. G. H. Baldwin, M.D. Hoogerland, S.J. Buckman, T.J. Senden, T.E. Sheridan, R.W.Boswell, J. Vac. Sci. Technol. B 16 (6), (1998)

[7] P. Engels, S.Salewski, H. Levsen, K. Sengstock, W. Ertmer, Appl. Phys. B. 69, 407-412, (1999)

[8] K.S. Johnson, K.K. Berggren, A.Black, C.T. Black, A.P.Chu, N.H. Dekker, D.C. Ralph, J.H. Thywissen, R. Younkin, M. Tinkham, M. Prentiss, G.M. Whitesides, Appl. Phys. Lett. 69 (18), (1996)

[9] S..J. Rehse, A.D. Glueck, S.A. Lee, A.B. Goulakov, C.S. Menoni, D.C. Ralph, K.S. Johnson, M. Prentiss, Appl. Phys. Lett. 71 (10), (1997)

[10] M. Baker, A.J. Palmer, R.T. Sang, Meas. Sci. Tech. (submitted, 2002)

[11] . C. D. Bain, E. B. Troughton, Y. Tao, J. Evall, G.M. Whitesides, R.G. Nuzzo, J. Am. Chem. Soc 111, 321-335, (1989)

[12] F.L. McCrackin, E. Passaglia, R.R. Stromberg, H.L. Steinberg, J. Res. Natl. Inst. Stand. A 67A (4), 363-377, (1963)

[13] Y. Xia, X. Zhao, E. Kim, G.M. Whitesides, Chem. Mater. 7, 2332-2337 (1995) 